Oort: If one supposes that the expansion velocities observed in the central region are caused by an explosion at the centre, it is necessary to assume that a process of ejection of large amounts of matter from the nucleus has been going on over a long interval of time. While the matter pushing out the $3-\mathrm{kpc}$ arm must have left the nucleus some 50 million years ago, the highvelocity matter with positive velocities observed close to the centre must have started only a few million years ago.

I would like to ask Dr. Parijsky whether he really believes that the emission in the southern part of what he calls a ring is further than $3 \mathrm{kpc}$ from the centre. From the appearance of the structure near the direction of the centre, this seems rather improbable.

Parijsky: As far as the position of the sources is concerned it is indeed very strange if it is not a ring (or another combination of details) in the central region. Of course, it could also be a "window" in the 3-kpc arm.

\title{
42. RECENT OBSERVATIONS AT DWINGELOO OF THE CENTRAL REGION OF THE GALACTIC SYSTEM
}

\section{J. H. OoRT}

\section{Leiden Observatory}

The work reported on was done by G. W. Rougoor and is based on observations made with the Dwingeloo dish and the eight-channel receiver built by Professor Muller.

The longitude interval from -8 to $+22^{\circ}$ was studied in detail for four latitudes, at $b^{\mathrm{I}}=-0^{\circ} 5,-1: 0,-1.5$, and $-2^{\circ} 0\left(b^{\mathrm{II}}=\right.$ about $+0^{\circ} 9,+0^{\circ} .4,-0^{\circ} 1$, and $\left.-0^{\circ} 6\right)$. The maximum intensity is in general found at $b^{\mathrm{I}}=-1.5$ (Fig. 1). This level gives the clearest picture of the gas in the small, rapidly rotating disk. Some other features stand out better at other latitude levels. At $b^{\mathrm{I}}=-1.0$ one can trace a rather continuous arm on the positive-velocity side from longitude $22^{\circ}$ down to $-5^{\circ}$. At the point where it passes behind Sgr A this arm appears to have a radial motion of $+135 \mathrm{~km} / \mathrm{sec}^{*}$ Its sudden ending at $l \mathrm{II} \sim-5.5$ suggests that at this point it is close to the outer edge of the disk. But its velocity at this point differs entirely from that of the gas within the disk.

The contour diagram for $b^{\mathrm{I}}=-2^{\circ} .0$ indicates two other features. At the positive-velocity side an "arm" is observed emerging from the general ridge of small velocities around $l \mathrm{II}=12^{\circ}$. The velocity of this arm rises steeply towards smaller longitudes up to a maximum of about $+230 \mathrm{~km} / \mathrm{sec}$ near $l \mathrm{II}=4^{\circ}$, where it may join up with the rapidly rotating disk to be described below. It is possible that this arm could be a continuation of the 3-kpc arm which is such a striking feature on the side of the negative velocities.

The second feature is seen at the negative velocities, crossing the longitude of the galactic centre. At this point it has a velocity of approximately $-100 \mathrm{~km} / \mathrm{sec}$. This may be due either to gas clouds near the edge of the rotating disk and moving away from the centre, or to part of a small expanding arm.

A possible arrangement of the arms and disk in the central region is shown in Figure 4 of paper 1.

*This, as well as all other velocities mentioned, is relative to the local standard of rest. 
There is a striking asymmetry in the appearance of the contour diagram at the side of positive longitudes and velocities and at the side of negative longitudes and velocities. Curiously, the total amounts of gas flowing away from the centre appear to be very much the same on the two sides; when considered on a sufficiently

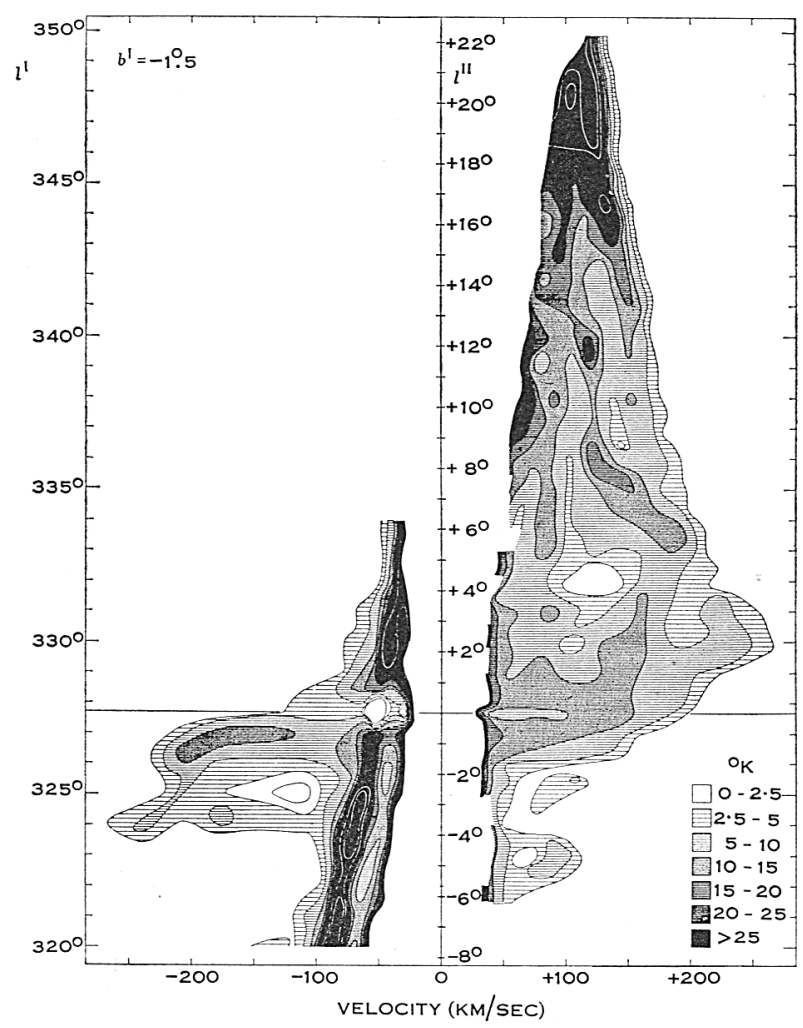

Fig. 1.-Contours of brightness temperature in the central region for a plane at $b^{\mathbf{I}}=-1.5$.

large scale they do not seem to vary much with longitude either (cf. Fig. 2(a)). The limits indicated in this Figure and in Figure 2(b) are velocities corrected for the motion of the Sun relative to the centre of the Galaxy. The numbers for $l \mathrm{II}<-8^{\circ}$ were estimated from unpublished data kindly supplied to us by Dr. Burke, and obtained with the 54-channel equipment at the Department of Terrestrial Magnetism in Washington, D.C.

Most of the radiation at negative velocities higher than those corresponding to the 3-kpc arm (cf. the negative-velocity wing between 0 and $-4^{\circ}$ longitude in Figure 1) seems to come from a disk of about 500 pc radius around the centre showing very high rotational velocities.* In order to get more precise measures around the nucleus, the region between -2 and $+2^{\circ}$ longitude and between latitudes -1 and $+1^{\circ}$ was covered by a dense grid of line profiles, spaced at 0.25 intervals in either coordinate.

*Rougoor, G. W., and Oort, J. H. (1960).-Proc. Nat. Acad. Sci. (Washington) 46: 1-13. 
At positive velocities the picture is confused because we have rather highvelocity expanding matter superimposed on the "disk". In order to investigate whether the rotating disk is also present at the positive-longitude side of the centre, only those velocities were considered which were higher than $150 \mathrm{~km} / \mathrm{sec}$. The resulting numbers of atoms per $\mathrm{cm}^{2}$ are plotted in Figure 2(b); they refer to observations at $b^{\mathrm{II}}= \pm 0.50$ and \pm 0.75 , the lowest latitudes being omitted in order to avoid the principal part of effects of absorption in the Sagittarius source. It may be observed that the two sides show a striking resemblance, and particularly that on
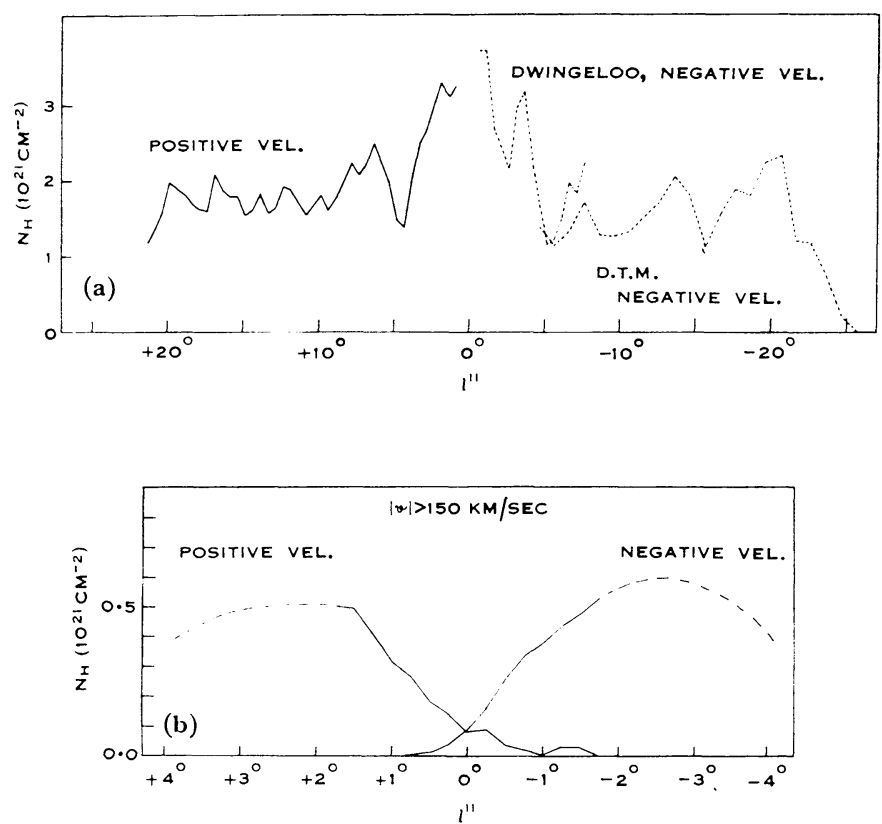

Fig. 2.-Variation with longitude of the amount of expanding matter. (a) Number of HI atoms in the line of sight. (Velocities between $\pm 40 \mathrm{~km} / \mathrm{sec}$ have been left out.) (b) Disk $\left(b^{\mathrm{II}}= \pm 0^{\circ} .50\right.$ and $\left.\pm 0^{\circ} .75.\right)$

both sides there is a drop to practically zero right at the longitude of the centre. This would appear to be a strong indication that we are dealing with a nuclear disk. It may well be that the "disk" is in reality quite irregular and patchy, as has been indicated by Kerr. The mean latitude of the disk is $b^{\mathrm{II}}=-0^{\circ} 03$, its mean thickness is about $50 \mathrm{pc}$, but is probably still smaller near the centre.

In the 3-kpc arm a special study was made of the density distribution in a direction perpendicular to the galactic plane, and also of the internal velocities. Both studies were confined to the longitude interval from $-7: 7$ to $-2: 7$; in latitude the

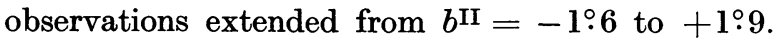

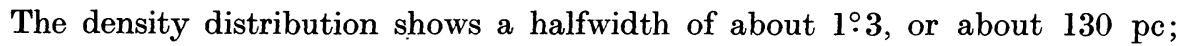
it has a rather sharp peak near $b^{\mathrm{II}}=+0^{\circ} 1$ superimposed on a broader component. Some of the pertinent data are shown in Table 1 . 
It will be seen that the random motions show a minimum around $b^{\mathrm{II}}=-0.4$ and a maximum at the highest positive latitudes. The systematic motions show a linear decrease with increasing latitude from -0.6 to +099 . Outside this interval they indicate a more irregular pattern.

Phenomena observed in some galaxies, and most convincingly in M82, indicate that explosions involving masses of the order of millions of solar masses sometimes occur in galactic nuclei. We have considered the possibility that the expanding features in the central region may be due to the explosion of such a large mass in the galactic centre. In order to explain the radial motions observed in the 3 -kpc arm,

TABLE 1

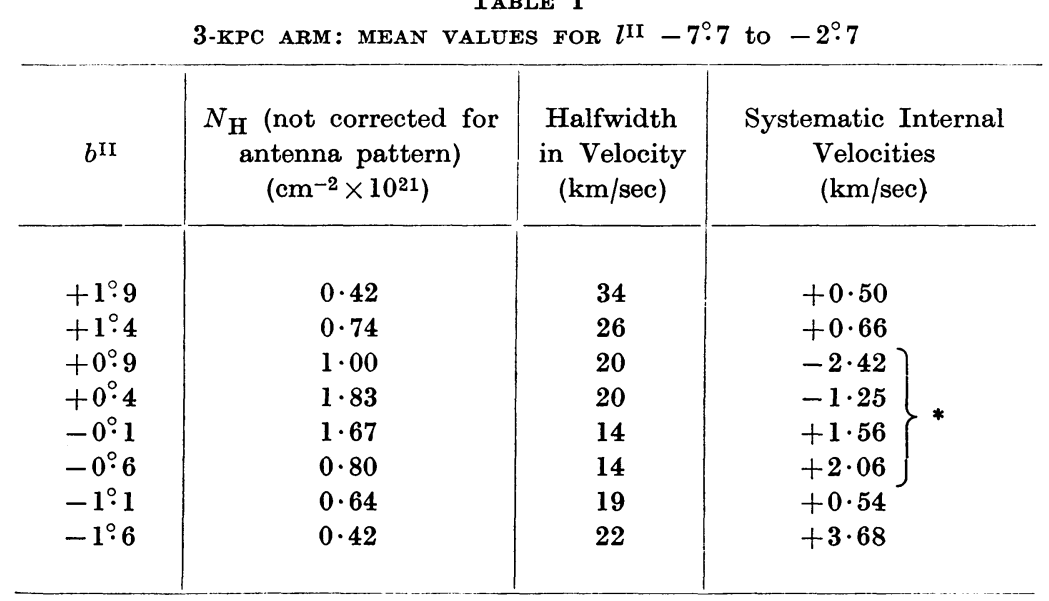

* Linear relation for the inner part, indicating "rolling" motion.

the exploded gas should have had a mass of the order of $10^{7}$ solar masses and an initial velocity of a few thousand $\mathrm{km} / \mathrm{sec}$. The $3-\mathrm{kpc}$ arm should then consist largely of gas previously collected in the galactic disk within $3 \mathrm{kpc}$ from the centre and swept up by the high-velocity shell. The explosion by which the 3-kpc arm was pushed out should have occurred about 30 or 40 million years ago. The much faster moving material behind the centre could hardly have started more than 5 million years ago. Therefore, if super-explosions of this kind are responsible for the observed phenomena, they must have continued over a period of at least 30 million years.

\section{Discussion}

de Vaucouleurs: What is the distance from the centre to the tangent point of the 3-kpc arm at the positive-longitude side as seen from here?

Oort: Perhaps 1-1 $\frac{1}{2} \mathrm{kpc}$. This is very uncertain since this region itself cannot be observed, as it is near zero velocity. We start to see it again only when the expansion velocity exceeds the rotational velocity.

de Vaucouleurs: I wish to point out that it is difficult to reconcile a two-armed inner structure as drawn on the board with a multi-armed structure in the outer parts. Our map or model of the Galaxy must agree with observed structure in other galaxies.

Oort: Information about the $3 \mathrm{kpc}$ arm is rather definite, but some of the rest of the suggestions of structure I gave for this region are highly hypothetical. 
Lindblad: I should like to ask if an alternative to the spiral structure has been contemplated, for instance, a sort of elliptical ring with apsidal line inclined towards the direction of the Sun.

Oort: One difficulty would be that some of the very prominent features such as the high positive velocity at negative longitudes are not consistent with such an analysis.

Perek: Is it possible to assume that the two spiral arms are symmetrical or is there any indication of asymmetry?

Oort: There seems to be a higher expansion velocity in the matter behind the centre than in the arm nearest to us.

Aller: Do the data exclude the existence of a bar at the centre of the Galaxy?

Oort: No, but the structure cannot be symmetrical on both sides; if a bar is present, it would have to be asymmetrical.

\section{HYDROGEN MOTIONS IN THE CENTRAL REGION OF THE GALAXY}

\section{B. F. Burke and M. A. Tuve}

Carnegie Institution of Washington, Department of Terrestrial Magnetism

The 21-cm hydrogen radiation near the galactic plane exhibits strong deviation from circular motion over longitudes within $30^{\circ}$ or so of the galactic centre. In order to extend the observations first made by Rougoor and Oort, ${ }^{*}$ the Carnegie multichannel $\mathrm{H}$-line spectrograph was used with our 60 -foot radio telescope at Derwood, Maryland, in order to confirm the Leiden work and extend observations to more southern longitudes. Cross-sections extending $\pm 2^{\circ}$ in latitude were taken every $2^{\circ}$ in longitude from $l \mathrm{II}=-26^{\circ} .8$ to $l \mathrm{II}=+2^{\circ} 2$ and a series of points along the galactic equator were taken every $1^{\circ}$ over the same range. Effort has been concentrated on the southern extension of the observations, which agree well with the Leiden observations over the section in common, but which carry the observations nearly $20^{\circ}$ farther south in longitude.

The observations in the plane are summarized in Figures 1 and 2, which show, respectively, the run of the hydrogen maxima and the temperature contours as a function of $l^{\mathrm{I}}$ and $V_{r}$. The " $3 \cdot 5$-kpc expanding arm" dominates the diagrams from the centre to about $l^{\mathrm{I}}=320^{\circ}\left(l^{\mathrm{II}}=-7 \cdot 8\right)$, but farther south the line profiles are much more complicated, showing three or four peaks as a general rule. The " $3 \cdot 5$ kpc arm" appears, in Figure 1, to break into two parts at $l \mathrm{I}=320^{\circ}$, although the possibility exists that two distinct arms are being observed that just happen to merge in velocity at this point. At $l \mathrm{I}=315^{\circ}$, the " $3 \cdot 5$ - $\mathrm{kpc}$ arm" also appears to undergo a change, and examination of the records indicates that there is still a weak extension (not shown in Figure 1) to about $l^{\mathrm{I}}=312^{\circ}$ of a feature whose velocity varies linearly with $l$. The weakest contour line in Figure 2 shows the remnant clearly. Figure 3 shows a series of cross-sections in $b$ that also show the highvelocity extension, which no longer lies in the plane at $l^{\mathrm{I}}=312^{\circ}\left(l^{\mathrm{II}}=345^{\circ} \cdot 3\right)$ but is clearly defined, with a velocity of nearly $160 \mathrm{~km} / \mathrm{sec}$.

*Rougoor, G. W., and Oort, J. H. (1960).-Proc. Nat. Acad. Sci. (Washington) 46: 1-13. 